\title{
Miniaturized optical chemosensor for flow-based assays
}

\author{
Marta Pokrzywnicka • David J. Cocovi-Solberg • \\ Manuel Miró • Víctor Cerdà • Robert Koncki • \\ Lukasz Tymecki
}

Received: 10 September 2010 /Revised: 22 October 2010 /Accepted: 24 October 2010 /Published online: 20 November 2010

(C) The Author(s) 2010. This article is published with open access at Springerlink.com

\begin{abstract}
A cost-effective, highly compact, and versatile optoelectronic device constructed of two ordinary light emitting diodes compatible with optosensing films has been developed. This fibreless device containing chemoreceptor, semiconductor light source, and detector integrated in a miniaturized flow-through cell of low microliter internal volume works as a complete photometric chemical sensor suitable for detection in flow analysis. The operation of the developed device under nonstationary programmableflow conditions offered by sequential injection analysis has been demonstrated using Prussian Blue film as a model optical chemoreceptor. The unique spectroelectrochemical properties of the sensing material enable its use for optical sensing of redox species, whereby ascorbic acid and hydrogen peroxide have been chosen as model analytes. The reported SI-sensor system features fast and reproducible determination of both analytes in the submillimolar range of concentrations. The construction concept demonstrated in this work can be easily applied to other kinds of optical sensors based on absorbance sensing films.
\end{abstract}

Electronic supplementary material The online version of this article (doi:10.1007/s00216-010-4384-2) contains supplementary material, which is available to authorized users.

M. Pokrzywnicka $\cdot$ R. Koncki $\cdot$ Ł. Tymecki $(\bowtie)$

Department of Chemistry, University of Warsaw,

Pasteura 1,

02-093 Warsaw, Poland

e-mail: luktym@chem.uw.edu.pl

D. J. Cocovi-Solberg $\cdot$ M. Miró $(\bowtie) \cdot$ V. Cerdà

Department of Chemistry, University of the Balearic Islands,

Carretera de Valldemosa $\mathrm{km} \mathrm{7.5,}$

07122 Palma de Mallorca, Spain

e-mail: manuel.miro@uib.es
Keywords Sensor Sequential injection analysis . Optosensing $\cdot$ Light emitting diodes $\cdot$ Prussian blue

\section{Introduction}

Photometry should be regarded as the most often applied detection technique in the analytical practice because almost all analytes are colored or could be selectively converted into colored species for easy detection using optical methods. Many of them have been transferred to the sensor format. A vast number of optical sensors and biosensors reported and reviewed in the analytical literature [1-5] foster the detection of a broad spectrum of analytes ranging from simple molecules (including gases, $\mathrm{pH}$, anions and cations), to organic compounds encompassing low molecular-weight sugars, metabolites and toxins and large biomolecules as well including peptides, proteins, enzymes, immunocompounds, DNA etc. The operation of the most popular fiber-optic chemical sensors (FOCS) is based on analyte-dependent changes of optical properties such as absorbance, diffuse reflectance or fluorescence of their receptor part [1-5]. The chemical sensing and signal processing capability of the chemo-optical interface is achieved by the incorporation of a given reagent. The reagent layer usually contains a dye (e.g., chromoionophores or acid-base indicators) which reversibly reacts with the analyte contained in the sample. The main role of the optical fibers is the transmission of light from the external light source to the sensing layer and from the sensing layer to the detection system. The advantages of such assembly are the ability of remote sensing and the probe format of FOCS. However, the use of optical sensors in flow measurements demands additional adaptation of FOCS to 
a flow-through format or the FOCS mounting into dedicated flow cells of optimized geometry.

The main goal of this work is the development of a fibreless, highly compact flow-through optosensing device containing the three main components of optical chemosensors, that is, an immobilized chemical that optically responds to the analyte (chemoreceptor), compatible light source and optical detector, in a miniaturized format using extremely low-budget optoelectronics (light emitting diodes, LEDs) and signal transducing/recording equipment (ordinary voltmeter). The device is based on the concept of paired-emitter-detector-diode (PEDD) developed for batchwise analytical measurements by Diamond's group [6]. According to this concept, two LEDs are paired together, one used in a conventional way as a light source whereas the second one as a light detector, resulting in a complete absorbance detector. Tymecki et al. [7] demonstrated that the electromotive force generated by PEDDs can be exploited as analytical signal. Hence, the coupling of PEDDs to $\mathrm{pH}$ meters [7] or ordinary voltmeters [8] leads to integrated, low-cost photometers. As demonstrated previously [9], ordinary, low-budget multimeters can play a double role, that is, working as an electric source for detector powering and simultaneously as a signal-reading instrument. The reported PEDD-based devices have been applied so far to batchwise (bio)analytical cuvette measurements [9-12] with potential use as flow-through cells in flow injection analysis [7-13] and chromatographic separations [14-17].

There has been however little effort as of yet towards the integration of optoelectronic components with chemosensitive layers for design of dedicated optrodes. The sensoric operation of the integrated device is the result of photometrically monitored changes in the optical properties of the chemoreceptor. A survey of literature revealed two recent examples of the usage of PEDDs in combination with sensitive films, yet merely applied to textile-based wearable [18] and gas [19] $\mathrm{pH}$ sensing. To the best of our knowledge, however, no flow-through PEDD-optrode for assays of aqueous samples in flow analysis or meso/ microfluidic devices has been reported so far.

In this communication, a universal device for flowthrough chemical optosensing is presented and characterized. The integrated chemosensor features four assets: (a) designed as a dedicated detector for flow analysis and related approaches thereof including microchip-based platforms; (b) compatible with optically chemosensitive materials obtained from conventional large-scale methodologies, that is, physical or chemical deposition onto thin transparent plastic films like polyester foil, (c) miniaturized and tailorable to a hand-held fashion and (d) cost-effective and easy to fabricate.

\section{Materials and methods}

Chemicals and reagents

4-(Pyrrol-1-yl)benzoic acid (Pyr-BAc) was obtained from Aldrich (Germany). All other reagents used in this work were of analytical grade. All solutions used in this work were prepared using doubly distilled water.

\section{Sensing film preparation}

The Prussian Blue (PB) film used in this study as an optochemically sensitive component of the sensor [20] was obtained by soaking a $100 \mu \mathrm{m}$-thick transparent plastic foil (Lumocolor ${ }^{\circledR}$ universal photocopy film, Staedtler ${ }^{\circledR}$,) into a solution of $0.1 \mathrm{~mol} \mathrm{~L}^{-1} \mathrm{~K}_{3} \mathrm{Fe}(\mathrm{CN})_{6}$ in $1 \mathrm{~mol} \mathrm{~L}^{-1} \mathrm{HCl}$ saturated with Pyr-BAc as additive. In a strongly acidic environment the hexacyanoferrate complex slowly decomposes forming the PB film (so-called "insoluble form", that is, $\left(\mathrm{Fe}_{4}\left[\mathrm{Fe}(\mathrm{CN})_{6}\right]_{3}\right)$. Oxidative polymerization of Pyr-BAc also takes place, leading to the formation of a mixed inorganic-organic composite material. The chemical process for film deposition is initiated and accelerated by UV irradiation. The preparation protocol and mechanism of the nonelectrochemical deposition of the composite material on nonconducting supports have been reported in detail elsewhere [21].

Apparatus and analytical procedure

The construction of the sensor device presented in this work capitalizes on LEDs (product no. OSHR53E1A2M from Optosupply, Hongkong). The LED emitter was powered with a constant-current source consisting of a simple home-made circuitry with light intensity modulation. The electromotive force (EMF) signal generated by the sensor was recorded using an ordinary multimeter from Voltcraft (model VC820, Germany) connected via an RS232 interface to data storage PC. The use of low internal resistance voltmeter offers significant enhancement of sensitivity [8-10].

The miniaturized flow-through sensor was integrated in a sequential injection (SI) fluid handling system composed of a 1-mL syringe pump (Micro BU 2030, Crison Alella, Spain) working as a pressure-driven liquid-driver, an eightport multiposition selection valve (Crison) and a 45 position rack autosampler (Micro Sampler Crison). The flow network fosters automatic metering of well-defined volumes of solutions at will and delivery to the flow-through optrode. The software package AutoAnalysis 5.0 (Sciware, Palma de Mallorca) based on dynamic link libraries was employed for automated control of the instrumentation including the 
multimeter and data acquisition as well. The flow manifold is schematically illustrated in Fig. 1a.

The analytical procedure involves the sequential aspiration into the holding coil $(0.8 \mathrm{~mm}$ i.d., $1.5 \mathrm{~m}$-long $)$ of $200 \mu \mathrm{L}$ of regenerator solution and $200 \mu \mathrm{L}$ of sample separated by $50 \mu \mathrm{L}$ carrier $\left(0.2 \mathrm{~mol} \mathrm{~L}^{-1} \mathrm{KH}_{2} \mathrm{PO}_{4} / \mathrm{K}_{2} \mathrm{HPO}_{4}\right.$, $\mathrm{pH}$ 7) working as segment spacer. Thereafter, the stacked segments were delivered by pressure-driven flow to the optosensor at $\leq 0.8 \mathrm{~mL} \mathrm{~min}^{-1}$, impinging on the chemoreceptor surface to generate the transient signal, which resembles the typical readout in flow injection assays, followed by backward flowing of liquids to waste. The sequence of segments delivered to the flow-through sensor cell and flow profile are schematically shown in Fig. 1a. The SI setup incorporating the PEDD sensor has been proven suitable for implementation of automated assays of both reducing and oxidizing species without manifold reconfiguration (see below) provided that suitable regeneration/ conditioning solutions (namely, $10 \mathrm{mmol} \mathrm{L} \mathrm{L}^{-1} \mathrm{H}_{2} \mathrm{O}_{2}$ in
$0.3 \mathrm{~mol} \mathrm{~L}^{-1} \mathrm{KCl}$ at $\mathrm{pH} 2$ for reductants and $10 \mathrm{mmol} \mathrm{L}^{-1}$ ascorbic acid in $0.2 \mathrm{~mol} \mathrm{~L}^{-1} \mathrm{KH}_{2} \mathrm{PO}_{4} / \mathrm{K}_{2} \mathrm{HPO}_{4}$ buffer at $\mathrm{pH}$ 7 for oxidants) are placed at the peripheral ports of the multiposition selection valve.

\section{Results and discussion}

Flow-through sensor

For construction of the optosensing device, two identical and commercially available low-budget LEDs $(5 \mathrm{~mm}$ diameter, with flat front) have been used. The sketch and close-up of the prototype sensor are illustrated in Fig. 1b, c and d. The flow inlet and outlet are drilled through the plastic body of the LED detector (see Fig. 1c and d), generating the socalled flow-cell within LED [22]. The second LED operating as a light emitter is utilized without any mechanical modification. A piece of plastic foil bearing
A

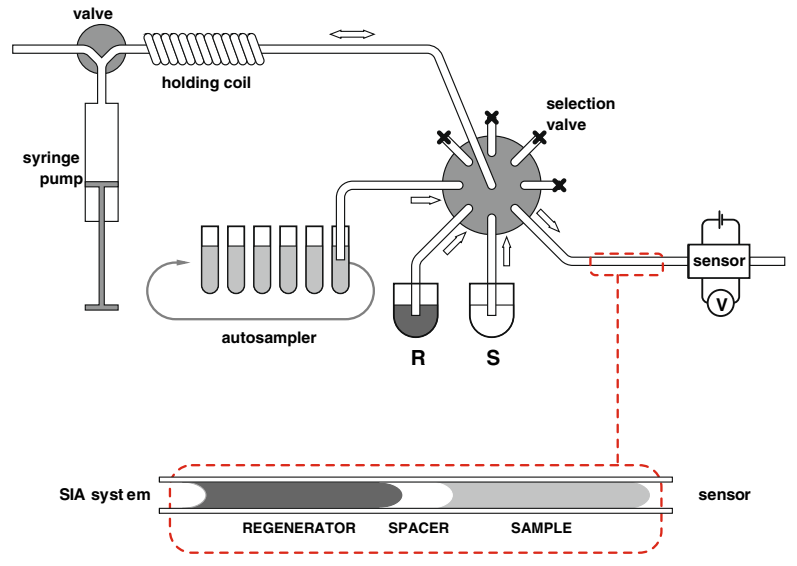

B

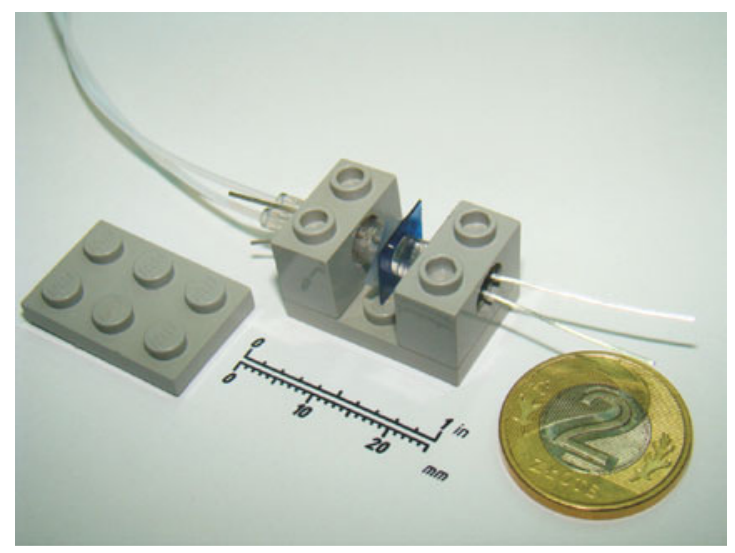

C
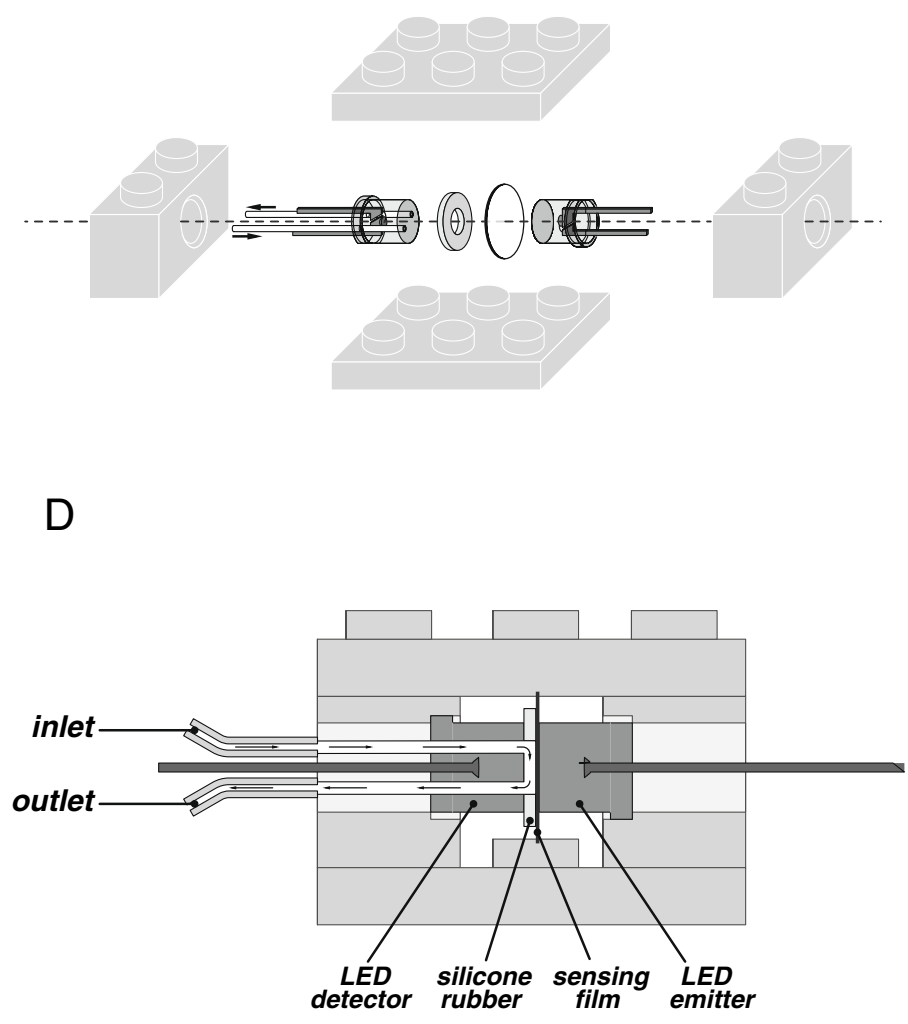

Fig. 1 Schematic illustration of A PEDD-based chemosensor integrated in a sequential injection (SI) manifold, B close-up of the assembled optrode, $\mathbf{C}$ expanded components of the sensor and $\mathbf{D}$ magnified sketch of the flow-through optosensor 
the sensing film is located on the top of the flat bulb of the LED emitter, acting as essential component of the flowthough cell. An elastic o-ring made of silicone rubber is located between the LED detector and film and defines the thickness as well as the inner volume of the resulting optical cell. The sealing of the flow-through cell is accomplished by clamping of LEDs with Lego bricks.

Contrary to PEDD-based photometric detectors for measurement of the absorbance in solution [6-17], the optical pathlength in the PEDD-based sensor herein presented should be minimized, because the device is dedicated to the optosensing of the reactive film. Besides, the miniaturized detector should be designed to provide maximum interaction of the radiation beam from the LED emitter with the foil sensing area, and prevent light loss, aimed at increasing the signal-to-noise ratio. The architecture of the developed PEDD device illustrated in Fig. 1 fulfills the above requirements: The optical pathlength through the solution (determined by the o-ring thickness) is merely $250 \mu \mathrm{m}$. The area of the sensor film contacting with solution is $0.07 \mathrm{~cm}^{2}$ equivalent to a $3-\mathrm{mm}$ diameter sensing microzone. Thus, the total internal volume of the developed flow-through cell housing the chemosensor layer is ca $1.7 \mu \mathrm{L}$.

The selection of the pair of LEDs for the PEDD is dictated by the optical properties of the sensing film. In this study the model sensing film is made of $\mathrm{PB}$, featuring deep blue color as a result of the wide absorption band at wavelengths $>600 \mathrm{~nm}$. The absorbance spectrum of this compound is compatible with the emission spectrum of red LEDs. Thus, a red LED has been selected as a light emitter. An LED can be merely used as a detector for light of equal or higher energy (low wavelengths) than the light emitted by itself [7, 23]. Therefore, for the construction of PEDD optically compatible with PB-based sensing film, two identical red LEDs have been herein employed.

It is worth to notice that the red LED with drilled flow channels (see Fig. 1c and d) playing the role of detector is light sensitive in the entire visible spectrum $(\lambda \leq 650 \mathrm{~nm})$ $[7,23]$. This denotes the universal nature of the miniature PEDD-based flow-through sensor, which might be furnished with a wide variety of sensing films and LED emitters operating in the visible range. As the flow-through chemosensor solely involves mechanical drilling of the LED detector body, construction of any other type of PEDD-based sensor is readily accomplishable by resorting to unmodified LED emitters optically compatible with the sensing film.

The optical sensitivity of the reported sensor exploits the unique spectroelectrochemical properties of PB $[20,21$, 24]. In the sensing scheme herein proposed, PB plays a double role: first as immobilized reagent (oxidant), and second as an optical sensoric receptor. Strong reductants cause discoloration of the sensing material. Reduction of
PB by ascorbic acid, used in this study as a model analyte, yields Prussian White ( $\mathrm{PW}$, namely, $\mathrm{K}_{2} \mathrm{FeFe}(\mathrm{CN})_{6}$ ). The transparent and colorless PW film does not absorb in the Vis-NIR range of spectrum. The PW film can be easily reoxidized to $\mathrm{PB}$ using various oxidants. Hence, hydrogen peroxide was used as a model regeneration agent. This reversible optical sensing scheme based on the $\mathrm{PB} / \mathrm{PW}$ redox system was integrated with optically compatible LEDs to demonstrate the figures of merit of the sensor.

In the course of preliminary experiments (data not shown), replicate measurements of ascorbic acid using concentrations of analyte and regenerator at the $10 \mathrm{mmol} \mathrm{L}^{-1}$ level both were weakly reproducible. It was found, that the first three to six peaks are less repeatable as a consequence of the usage of a freshly prepared PB film. As mentioned above, the freshly prepared PB film is composed of the so-called "insoluble PB". Fortunately, after a few red-ox cycles the sensing film material is converted into a more reactive, so-called soluble form $\left(\mathrm{KFeFe}(\mathrm{CN})_{6}\right)$ [20] and then consecutive measurements are nicely repeatable. The relative standard deviation for three preconditioned $\mathrm{PB}$ films of slightly different thickness bearing the $\mathrm{KFe}^{\mathrm{III}} \mathrm{Fe}^{\mathrm{II}}(\mathrm{CN})_{6} / \mathrm{K}_{2} \mathrm{Fe}^{\mathrm{II}} \mathrm{Fe}^{\mathrm{II}}(\mathrm{CN})_{6}$ redox system varied from $0.7 \%$ to $1.2 \%(n=12$ peaks for each film/sensor tested). The need of pretreatment of the PB film by conditioning cycles of reduction and oxidation before use for the first time is in agreement with earlier findings [21].

\section{Reductant sensing}

An important feature in PEDD sensor operation is the ability of tuning optrode's sensitivity by modulation of the current powering the LED emitter [8]. This PEDD attribute is well illustrated in Fig. 2 (top), where the readouts for a single standard of ascorbic acid $\left(10 \mathrm{mmol} \mathrm{L}^{-1}\right)$ are obtained at increasing currents from 0.7 to $2.0 \mathrm{~mA}$. This finding is significant in the general context of PEDD sensor development, because it offers the ability of optimization of PEDDbased procedures regardless of the transparency of the sensing layer, which is dictated by the thickness of the film.

Experimental results of sensor calibration performed at three different currents are compiled in Fig. 2 (middle). Both the baseline signal (related to light transmission through the chemosensitive layer) and optrode's sensitivity are markedly influenced by the LED emitter powering current. High currents $(\geq 1.4 \mathrm{~mA})$ yield calibration graphs with low sensitivity (see Fig. 2 top) because the enhancement effect attainable with the low-resistance EMF-reading instrument is suppressed. Nearly identical results could be obtained using $\mathrm{pH}$ meters offering almost currentless conditions for EMF measurement [7, 8]. As to smaller PEDD powering currents $(<0.7 \mathrm{~mA})$, which lead to a weak illumination of the LED detector, a low sensitivity is observed because the effect of LED detector discharging by 
Fig. 2 Analytical performance of the SI-PEDD-chemosensor for reductant detection. The effect of PEDD powering current (values given at the SIgrams) on the sensor response for $10 \mathrm{mmol} \mathrm{L}^{-1}$ ascorbic acid and the corresponding current-signal dependence (top). Readouts for ascorbic acid determination using the

SI-PEDD chemosensor powered at three different currents (given at the SIgrams) and

corresponding calibration graphs (middle). Readouts for ascorbic acid determination at three different reaction times (given at the SIgrams) and corresponding calibration graphs (bottom)
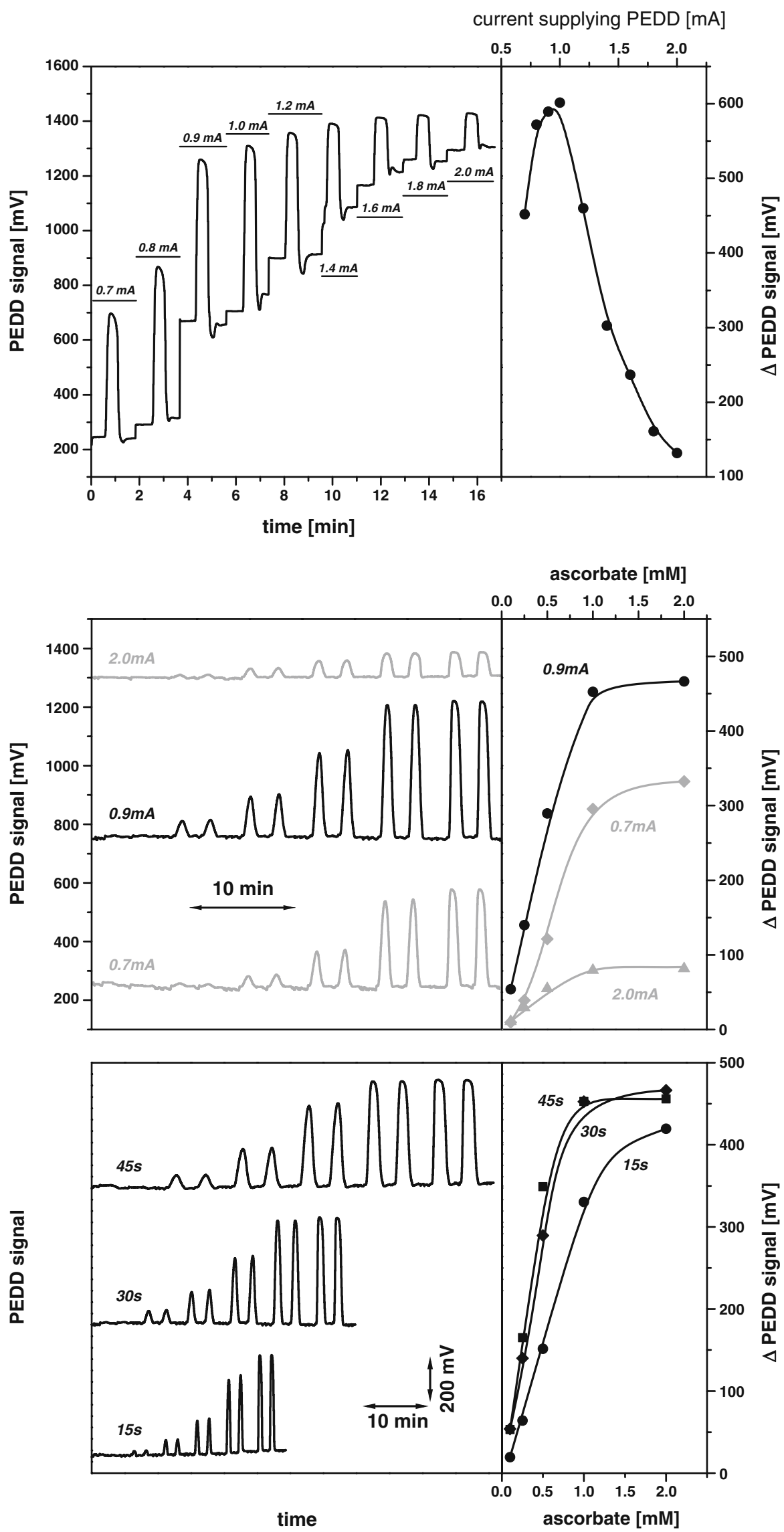
the signal-reading-instrument is stronger than the LED charging by illumination [8]. Intermediate PEDD powering currents $(0.7-1.0 \mathrm{~mA})$ are appropriate for monitoring of the chemoreceptor (Fig. 2, top and middle) because the above opposed processes are now of the same magnitude. A linear regression equation within the concentration range of 0.1 to $0.5 \mathrm{mM}$ ascorbic acid (sensitivity, $574 \mathrm{mV} / \mathrm{mM} ; R^{2}=0.999$ ) was found at $0.9 \mathrm{~mA}$ (see Fig. 2 middle) with a detection limit (LOD) of $20 \mu \mathrm{M}$. Upper quantification limit might be extended up to $1.0 \mathrm{mM}$ by resorting to polynomial regression. The flow-through PEDD sensor features longterm stability with negligible LED corrosion and optrode leaking throughout consecutive assays. Sensor reusability is feasible yet daily recalibration is needed for reliable results because of eventual changes in optical properties of the chemoreceptor.

It is worth to notice that maximum currents commonly recommended for LED driving are more than one order of magnitude higher (ca. $30 \mathrm{~mA}$ ) than those applied in this work for operation of the optrode under maximum sensitivity (Fig. 2). This observation clearly indicates that the use of more sensitive photodiodes instead of LEDs as detectors in the developed measurement system has no practical use. In other words, the application of LEDs as light detectors suffices for effective operation of the devised PEDD sensor.

Yet another parameter significantly influencing the PEDD sensor response is the reaction time of the sample zone within the sensing film (Fig. 2 bottom). The higher the flow rate of sample delivery to the $\mathrm{PB} / \mathrm{PW}$ reactive microzone, the higher is the sample throughput, yet at the expenses of decreased sensitivity. In the case of PB sensing, however, saturation of the analytical signal is observed. This effect is caused by the "stoichiometric" nature of the analyte recognition process $[20,21]$ and limited PB load onto the film surface. The saturation of the signal, which determines the upper concentration level of the dynamic range, is caused by the entire depletion of the receptor phase. As shown in Fig. 2 (bottom), this phenomenon is distinctly observed at low flow rates $\left(\leq 0.4 \mathrm{~mL} \mathrm{~min}^{-1}\right)$ and high analyte concentrations $\left(\geq 1.0 \mathrm{mmol} \mathrm{L}{ }^{-1}\right)$, whereby sample delivery flow rates of $0.8 \mathrm{~mL} / \mathrm{min}$ were used in acid ascorbic assays.

Under the selected physical and chemical conditions, the PB-film-based PEDD-optrode was utilized for automatic assays of two ascorbic acid-containing formulated dosage forms, namely, Aspirin $+\mathrm{C}$ (Quimica Farmaceutica Bayer, SL, Sant Joan Despí, Barcelona) and Supradyn Active Multivitamin (Quimica Farmaceutica Bayer) with manufacturer's nominal values of $240 \mathrm{mg} / \mathrm{unit}$ and $60 \mathrm{mg} / \mathrm{unit}$, respectively. Drug formulations were dissolved or suspended in distilled water and analyzed with the flow-through optrode without further sample processing. Measurement trueness was estimated by resorting to iodometric titration [25] as a reference analytical method endorsed in pharmacopeias. No significant differences at the 0.05 significance level were encountered between the content in vitamin $\mathrm{C}$ as measured with the PEDD-optrode (viz., $223 \pm 3 \mathrm{mg} / \mathrm{unit}$ and $45 \pm$ $5 \mathrm{mg} / \mathrm{unit}$, expressed as mean \pm standard deviation, for aspirin $+\mathrm{C}$ and Supradyn Active Multivitamin, respectively) and the titrimetric reference method (viz., $226 \pm 1 \mathrm{mg} / \mathrm{unit}$ and $48 \pm 2 \mathrm{mg} / \mathrm{unit}$ for aspirin $+\mathrm{C}$ and Supradyn Active Multivitamin, respectively) by resorting to a $t$ test for comparison of two experimental means [26]. A striking feature of the miniaturized flow-through optical sensor herein developed is the feasibility of admitting colored and turbid samples, e.g., Supradyn Active Multivitamin water suspension, while rendering unbiased results.

\section{Oxidant sensing}

The reduced form of the sensing film (PW) is chemically unstable and easily regenerated to PB. However, the automated SI procedure fosters the in-line generation of this unstable redox state of the sensing layer reliably via pumping of a minute, well-defined plug of reducing agent of appropriate concentration (e.g., $200 \mu \mathrm{L}$ of $10 \mathrm{mmol} / \mathrm{L}$ ascorbic acid). In consequence, the in situ generated PW film can be used for optical sensing of oxidants. In such approach, the roles of oxidant and reductant are reversed: hydrogen peroxide is recognized by the PW-based sensing film as analyte, whereas ascorbic acid plays the role of sensor regenerator. As previously pinpointed for reductant detection (see Fig. 2 top), the current powering PEDD should be, for oxidant sensing, likewise adjusted within the range of $0.6-$ $2.0 \mathrm{~mA}$ (Electronic Supplementary Material Fig. S1 top). The current-signal characteristics for hydrogen peroxide sensing are almost the same. The minor variations in the current for maximal sensitivity are caused by slight differences in thickness of the sensor films used throughout this work.

Figure S1 illustrates the non-linear calibration graphs of the PEDD sensor for hydrogen peroxide within the concentration span of 0.1 to $2.0 \mathrm{mM}(\mathrm{LOD} \geq 40 \mu \mathrm{M})$ as obtained at varied PEDD powering currents (Electronic Supplementary Material Fig. S1 middle) and reaction times (Electronic Supplementary Material Fig. S1 bottom) using sample perfusion flow rates through the measurement system from 0.27 to $0.8 \mathrm{ml} / \mathrm{min}$. These effects are analogous to those earlier reported in this work for reductant detection (Fig. 2). The resulting SI peaks are however inverted because of decreased film transparency when sensing hydrogen peroxide (Electronic Supplementary Material Fig. S1). The slight differences in peak shapes as compared to reductant assays are a consequence of the electrocatalytic nature of hydrogen peroxide reduction by the $\mathrm{PB} / \mathrm{PW}$ system [20, 21]. Again, the optrode's sensitivity is 
mainly dictated by the PEDD powering current, whereas a decrease in flow rate improves sensor detection capability. In brief, the recordings and corresponding calibration graphs compiled in Electronic Supplementary Material Fig. S1 (middle and bottom) clearly demonstrate that the developed flow-through optrode offers relatively fast detection of hydrogen peroxide in the submillimolar range. It is worth to mention that this mode of hydrogen peroxide sensing has potential use in bioassays exploiting oxidoreductases $[27,28]$.

\section{Conclusions}

The dedicated PEDD-based optrode herein reported for flowthrough chemical assays of aqueous samples features several advantages. It is an inexpensive sensor system enabling optosensing measurements using merely an ordinary voltmeter and two extremely low-cost LEDs. The total cost of the sensing device does not actually exceed 0.5 euro. Moreover, this miniaturized, and easily customized sensor is fully compatible with highly integrated systems capitalized on mesofluidic labon-a-valve and microfluidic lab-on-a-chip devices.

It should be stressed that the flow-through PEDD-optrode concept presented in this paper is of universal applicability. It can be readily expanded to a vast number of sensing schemes with chemosensitive materials, e.g., plasticized polymeric films containing immobilized indicator dyes or chromoionophores. Only careful selection is required for paired LEDs compatible with the optical properties of the sensing membrane along with the adjustment of the PEDD powering current for maximal optrode sensitivity as defined by the thickness and transparency of the sensing layer.

Acknowledgments These investigations were supported by research grant from the Polish Ministry of Science and Higher Education (Project no. N N204 029636) and Spanish Ministry of Science and Innovation (Project no. CTM2010-17214). Financial support from the Government of the Balearic Islands (Project no. AAEE0038/08) is also greatly acknowledged.

Open Access This article is distributed under the terms of the Creative Commons Attribution Noncommercial License which permits any noncommercial use, distribution, and reproduction in any medium, provided the original author(s) and source are credited.

\section{References}

1. Puyol M, Villuendas F, Domínguez C, Cadarso V, Llobera A, Salinas I, Garcés I, Alonso J (2005) Absorbance-Based Integrated Optical Sensors. In: Orellana G, Moreno-Bondi MC (eds) Series on chemical sensors and biosensors, vol. 3. Frontiers in chemical sensors, novel principles and techniques. Springer, Berlin

2. Wolfbeis OS (2006) Anal Chem 78:3859-3873

3. Wolfbeis OS (2008) Anal Chem 80:4269-4283

4. McDonagh C, Burke CS, MacCraith BD (2008) Chem Rev 108:400-422

5. Borisov SM, Wolfbeis OS (2008) Chem Rev 108:423-461

6. Lau KT, Baldwin S, O’Toole M, Shepherd R, Yerazunis WJ, Izuo S, Ueyama S, Diamond D (2006) Anal Chim Acta 557:111-116

7. Tymecki Ł, Pokrzywnicka M, Koncki R (2008) Analyst 133:1501-1504

8. Tymecki Ł, Koncki R (2009) Anal Chim Acta 639:73-77

9. Pokrzywnicka M, Koncki R, Tymecki Ł (2009) Chem Anal 54:427-435

10. Tymecki Ł, Brodacka L, Rozum B, Koncki R (2009) Analyst 134:1333-1337

11. Rozum B, Gajownik K, Tymecki Ł, Koncki R (2010) Anal Biochem 400:151-153

12. Tymecki Ł, Strzelak K, Koncki R (2009) Talanta 79:205-210

13. O'Toole M, Lau KT, Diamond D (2005) Talanta 66:1340-1344

14. Barron L, Nesterenko PN, Diamond D, O'Toole M, Lau KT, Paull B (2006) Anal Chim Acta 577:32-37

15. O'Toole M, Lau KT, Shazmann B, Shepherd R, Nesterenko PN, Paull B, Diamond D (2006) Analyst 131:938-943

16. Barron L, O'Toole M, Diamond D, Nesterenko PN, Paull B (2008) J Chromatogr A 1213:31-36

17. O'Toole M, Barron L, Shepherd R, Paull B, Nesterenko P, Diamond D (2009) Analyst 134:124-130

18. Morris D, Coyle S, Wu Y, Lau KT, Wallace G, Diamond D (2009) Sens Actuators B 139:231-236

19. O'Toole M, Shepherd R, Wallace GG, Diamond D (2009) Anal Chim Acta 652:308-314

20. Koncki R (2002) Crit Rev Anal Chem 32:79-96

21. Koncki R, Lenarczyk T, Głąb S (2000) Anal Chim Acta 424:27-35

22. Ugucione C, Cardoso AA (2007) Anal Bioanal Chem 389:16471650

23. Lau KT, Yerazunis WJ, Shepherd R, Diamond D (2006) Sens Actuators B 114:819-825

24. Lenarczuk T, Głąb S, Koncki R (2001) J Pharm Biomed Anal 26:163-169

25. Koncki R, Lenarczuk T, Głąb S (1999) Anal Chim Acta 379:69-74

26. Miller JN, Miller JC (2005) Statistics and chemometrics for analytical chemistry, 5th edn. Pearson, Harlow, England, pp 4145 , ch. 3

27. Koncki R, Lenarczuk T, Radomska A, Głąb S (2001) Analyst 126:1080-1084

28. Lenarczuk T, Wencel D, Głąb S, Koncki R (2001) Anal Chim Acta 447:23-32 